Draft version November 11, 2018

Preprint typeset using $\mathrm{LATE}_{\mathrm{E}} \mathrm{X}$ style emulateapj v. 5/2/11

\title{
SCALING OF THE GROWTH RATE OF MAGNETIC ISLANDS IN THE HELIOSHEATH
}

\author{
K. M. Schoeffler, J. F. Drake, and M. Swisdak \\ Institute for Research in Electronics and Applied Physics, University of Maryland, College Park, MD 20742-3511, USA \\ (Dated: November 11, 2018) \\ Draft version November 11, 2018
}

\begin{abstract}
Current sheets thinner than the ion inertial length are unstable to the tearing instability and will develop magnetic islands that grow due to magnetic reconnection. We investigate whether the growth of magnetic islands in a current sheet can continue indefinitely, or in the case of the heliosheath until reaching a neighboring current sheet, and at what rate the islands grow. We investigate the development and growth of magnetic islands using a particle-in-cell code, starting from particle noise. Performing a scaling of the growth of magnetic islands versus the system size, we find that the growth rate is independent of the system size up to the largest simulation we were able to complete. The islands are able to continue growing as long as they merge with each other and maintain a high aspect ratio. Otherwise there is not enough magnetic tension to sustain reconnection. When applied to the sectored magnetic fields in the heliosheath, we show that the islands can continue growing until they reach the sector width and do so in much less time than it takes for the islands to convect through the heliosheath.
\end{abstract}

At low latitude the solar wind is divided by the heliospheric current sheet into sectors of oppositely directed magnetic fields. The thickness of the current sheet, $\lambda$, is around $10,000 \mathrm{~km}$ (Smith 2001) at $1 \mathrm{AU}$, and the separation between each sector, or the sector width, is around 1AU. The sector width remains nearly constant, although increasingly variable, out to the termination shock (TS) at $\sim 90 \mathrm{AU}$, where the supersonic solar wind abruptly slows (Behannon et al. 1989; Burlaga et al. 2007). For the essentially collisionless environment of the solar wind $\lambda$ controls whether collisionless reconnection onsets. For $\lambda$ greater than the ion inertial scale, $d_{i}=c / \omega_{\mathrm{pi}}$, where $c$ is the speed of light and $\omega_{\mathrm{pi}}$ is the ion plasma frequency, reconnection via the collisionless tearing instability does not take place, while for $\lambda<d_{i}$ it does (Cassak et al. 2005; Yamada 2007). Just upstream of the TS where the ion density is $\sim 0.001 \mathrm{~cm}^{-3}, d_{i} \sim 7200 \mathrm{~km}$, which remains smaller than $\lambda$ based on measurements at 1AU. Thus, the absence of significant reconnection of the sector field upstream of the TS is consistent with models, although a definitive study of $\lambda$ upstream of the TS remains to be carried out. $\lambda$ downstream of the TS is predicted to be $2500 \mathrm{~km}$ based on measurements at $1 \mathrm{AU}$ and the shock compression, while $d_{i}$ is $4200 \mathrm{~km}$ based on ion density measurements of about $0.003 \mathrm{~cm}^{-3}$ (Richardson et al. 2008). Thus, downstream of the TS the current sheets should begin breaking up into magnetic islands.

The growth of islands in the finite plasma $\beta$ (the ratio of the plasma pressure to the magnetic pressure) heliosheath has been investigated in particle-incell simulations (Drake et al. 2010; Opher et al. 2011; Schoeffler et al. 2011). The predicted island length in the initial phase of reconnection is around $190 d_{i}$, much smaller than the sector width, $8900 d_{i}$, so it is uncertain whether islands will grow to the full sector width. Due to computational limitations it is not possible to realistically simulate the disparate ion inertial and sector scales. In this work, we perform a scaling study of the growth of islands, in which the inter-current sheet separation (equivalent to the sector width) varies, in order to understand what happens in the real system. Even our largest simulations have sector widths that are much smaller than in the actual sectored heliosheath.

The current sheets separating the sectored regions begin to form islands after crossing the TS. As the islands grow, the current sheets are convected toward the heliopause. The plasma flows outward at around $80 \mathrm{~km} \mathrm{~s}^{-1}$ and steadily decreases in speed for 20AU, at which point the radial flow remains close to zero (Krimigis et al. 2011). The important question which we seek to answer is whether the islands are able to expand to the sector width before the current sheet reaches the heliopause. If the islands expand to the sector width, there would be no more laminar field that can shield cosmic rays, and cosmic rays could percolate through the system consisting solely of islands. In addition, the full expansion of the islands would imply that acceleration mechanisms due to the growth of islands may play a significant role.

We simulate the growth of magnetic islands using a particle-in-cell code, p3d. The initial conditions consist of two oppositely directed current sheets in Harris equilibrium(Harris 1962), with a superimposed background density. The initial magnetic fields are in the $\hat{\mathbf{x}}$ direction, which corresponds to the azimuthal direction in the heliosheath. The current flows in the $\hat{\mathbf{z}}$ direction, which corresponds to the north south direction. The $\hat{\mathbf{y}}$ direction corresponds to the radial direction of the heliosheath. In the heliosheath the islands are predicted to be highly elongated due to the development of pressure anisotropy, and this elongation is dependent on both the ion-to-electron mass ratio and the electron temperature (Schoeffler et al. 2011). Typical simulations use a reduced mass ratio in order to reduce computational expenses, which produces much shorter islands than expected for the real system. We therefore use an enhanced temperature of the electrons in the background in order to form more realistic elongated islands. In the he- 


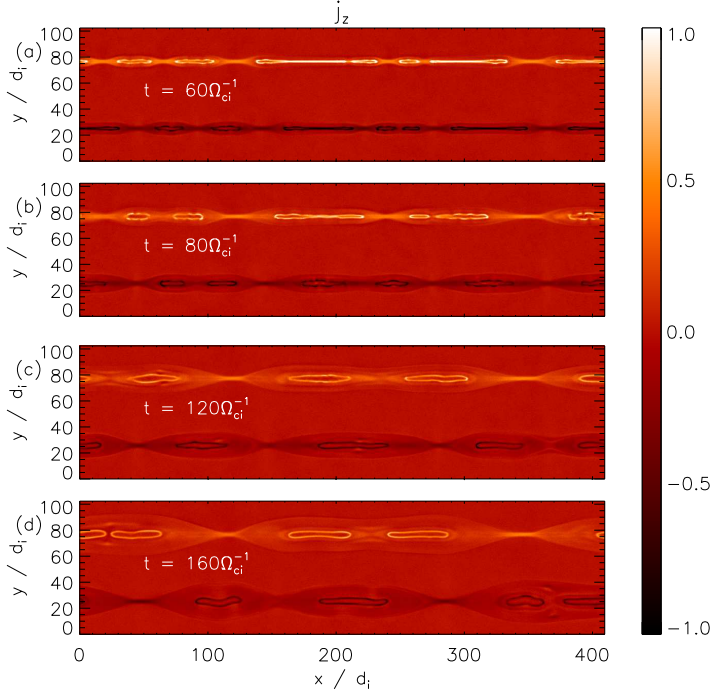

FIG. 1.- Out-of-plane current density, $j_{z}$, for $t=$ (a) $60 \Omega_{\mathrm{ci}}^{-1}$, (b) $80 \Omega_{\mathrm{ci}}^{-1}$, (c) $120 \Omega_{\mathrm{ci}}^{-1}$, and (d) $160 \Omega_{\mathrm{ci}}^{-1}$ For better contrast, all points with $\left|j_{z}\right|>1$ are assigned the colors shown for 1 or -1 .

liosheath nearly all of the pressure comes from the ions due to the pick-up ion population. In our simulation half of the pressure is in the ions and half is in the electrons. The $\beta$ of the plasma in the asymptotic field is 3 , which corresponds to a temperature in the heliosheath of $650,000 \mathrm{~K}$, based on a density, $n=0.003 \mathrm{~cm}^{-3}$, and a magnetic field, $B=0.15 \mathrm{nT}$. The ratio of the proton to electron mass in this simulation is 25 . We have run simulations that imply the reconnection rate is not sensitive to the value of the mass ratio, so our prediction should scale to the realistic mass ratio. The background temperature is 15 times the Harris sheet temperature of $0.25 m_{i} c_{A}^{2}$, where $m_{i}$ is the ion mass and $c_{A}$ is the Alfvén speed, based on the asymptotic magnetic field, $B_{0}$. The ratio of the speed of light to the Alfvén speed is 25. Each simulation has a grid scale resolution of $\Delta_{x}=\Delta_{y}=0.05 d_{i}$ and a time resolution of $d t=0.004 \Omega_{\text {ci }}^{-1}$, where $\Omega_{\text {ci }}$ is the ion cyclotron frequency. The half thickness of the current sheet is set to $w_{0}=0.5 d_{i}$ so that collisionless reconnection can begin from particle noise. We simulate a two-dimensional system. In three-dimensional systems islands form at different $z$ locations and grow along $z$, eventually stagnating likely due to interactions with other islands (Shav et al. 2003; Schreier et al. 2010). The significance of this effect in the heliosheath is unknown.

The time evolution of the largest of these simulations, with dimensions of $409.6 d_{i} \times 102.4 d_{i}$, can be seen in Figure 1] By $t=60 \Omega_{\mathrm{ci}}^{-1}$ the current sheet has broken into elongated magnetic islands as predicted in Schoeffler et al. (2011) (Figure 1(a)). The length of the islands is smaller than the separation between the two current sheets, so it is expected that they could not grow to the sector width since circular islands do not have tension to drive reconnection. However, as can be seen in the subsequent times (Figure [1(b)-(d)), the islands on a given current sheet begin to merge. Merging lengthens the islands which enables further growth until they approach the neighboring current sheet.

The islands found in the heliosheath, which are pre-

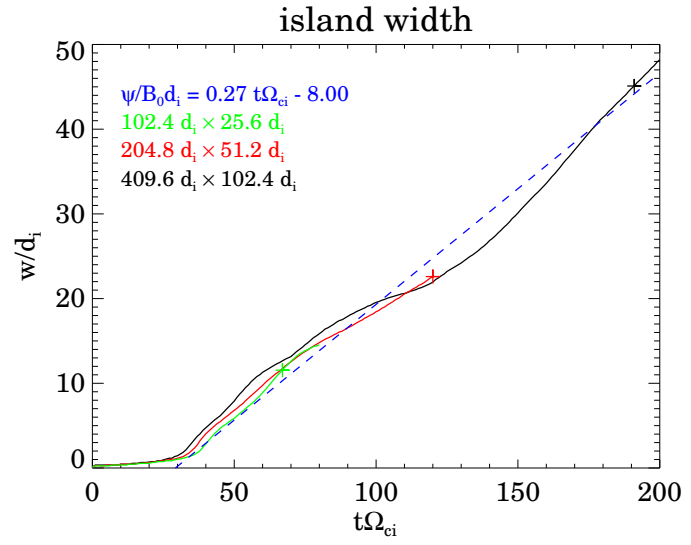

FIG. 2.- Width of the largest island in the lower of the two current sheets vs. time for simulations with dimensions of $102.4 d_{i} \times$ $25.6 d_{i}$ (green), 204.8di $\times 51.2 d_{i}$ (red), and $409.6 d_{i} \times 102.4 d_{i}$ (black). The plus signs denote the point where the island width reaches $44 \%$ of the system size and represent the time when the island begins to interact with the other current sheet. The blue dashed line is a line of best fit between these three points. The slope of this curve is the rate of growth at which the island expands, $0.12 c_{\mathrm{Ab}}$.

dicted to be much shorter than the current sheet separation, should in principle grow to the sector width as long as the islands are able to continue merging. The aspect ratio of our simulation box is 4 , which was sufficient for islands to continue merging up to the time that they begin interacting with the neighboring current sheet. We also carried out a simulation with aspect ratio 2 where the islands stopped merging when their length approached the system size. The islands saturated and stopped growing when there was no longer enough magnetic tension to maintain reconnection. The size of the heliosheath in the azimuthal direction greatly exceeds the sector width. Islands should therefore grow to the sector width. The next question is how long it takes for these islands to reach the sector width. In order to establish this time we carry out a scaling of the time required to grow to the sector width for system sizes $102.4 d_{i} \times 25.6 d_{i}$, $204.8 d_{i} \times 51.2 d_{i}$, and $409.6 d_{i} \times 102.4 d_{i}$.

To determine the size of the island we find the minimum of the flux function, $\psi$ where the magnetic field, $\mathbf{B}=\hat{\mathbf{z}} \times \nabla \psi(x, y)+B_{z}(x, y) \hat{\mathbf{z}}$, along the center of the lower initial current sheet at a particular time. This minimum corresponds to the most developed $x$-point. The upper current sheet has $x$-points at the maxima. The line of constant flux that crosses an $x$-point is known as the separatrix. The distance between the maximum and minimum $y$-locations of the separatrix is defined as the island width, $w$. Figure 2 shows the island width versus time for each of the simulations. We use the time when the island reaches $44 \%$ of the size of the box as a measure for when the island reaches the neighboring current sheet. At $50 \%$ the island begins to be affected by the presence of the neighboring current sheet. The bestfit line connecting the times when the islands reach the neighboring current sheet fits very well with the island width versus time for all the simulations.

The results of Figure 2 suggest that the islands grow at a nearly constant rate that is independent of the system size. Keeping in mind that this number is based on only three data points, using the slope of the best fit curve we can obtain an estimate for the rate of growth of the 


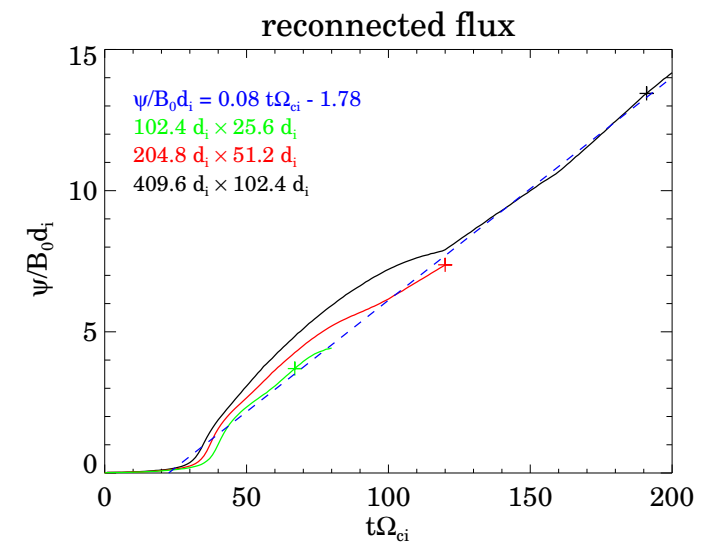

FIG. 3.- Reconnected flux in the lower of the two current sheets vs. time for simulations with dimensions of $102.4 d_{i} \times 25.6 d_{i}$ (green), $204.8 d_{i} \times 51.2 d_{i}(\mathrm{red})$, and $409.6 d_{i} \times 102.4 d_{i}$ (black). The plus signs denote the point where the island width reaches $44 \%$ of the system size and represent the time when the island begins to interact with the other current sheet. The blue dashed line is a line of best fit between these three points. The slope of this curve is the reconnection rate $0.079 B_{0} c_{A}$.

island of around $0.12 c_{A b}$, where $c_{A b}$ is the Alfvén speed based on the background density, which is distinct from the normalization of the code, $c_{A}$, which uses the peak density of the Harris equilibrium. If we extrapolate the growth rate to a very large system we can predict a time for the islands to reach the sector width. Based on a magnetic field, $B$, of $0.15 \mathrm{nT}$ and density, $n$, of $0.003 \mathrm{~cm}^{-3}$ the Alfvén speed just downstream of the TS is $60 \mathrm{~km} \mathrm{~s}^{-1}$. Using this speed for $c_{A b}$, and the sector width, $W=$ $0.25 \mathrm{AU}$, we obtain a growth time, $t_{g}$, of about 60 days, much less than the plasma convection time across the heliosheath.

$t_{g}=60$ days $\left(\frac{W}{0.25 \mathrm{AU}}\right)\left(\frac{n}{0.003 \mathrm{~cm}^{-3}}\right)^{1 / 2}\left(\frac{B}{0.15 \mathrm{nT}}\right)^{-1}$

Assuming the radial velocity of the solar wind inside the heliosheath is $70 \mathrm{~km} \mathrm{~s}^{-1}$ during island growth, this time corresponds to a distance of $2.5 \mathrm{AU}$ past the TS.
In addition to the rate of change in the island width being nearly constant, we find that the reconnection rate is also independent of system size. In Figure 3 we look at a similar plot to Figure 2, but of the reconnected flux. To find the reconnected flux we take the difference between the maximum and minimum of the flux function along the center of the initial current sheet. Based on the slope of the best fit curve, the reconnection rate was 0.079. Previous scalings of reconnection rate versus system size for conditions with lower plasma $\beta$, relevant to the Earth's magnetosphere and the 1AU solar wind, have shown comparable rates (Shay et al. 1999, 2007). However it is important to note that the reconnection rates shown in Shay et al. (1999, 2007) are associated with a steady state reconnection, as opposed to the rate shown here which includes the complicated dynamics of the merging process as well.

In the range of sizes simulated the rate of flux reconnection and the rate of island growth are nearly constant once reconnection begins. The growth rates are independent of the system size. It is reasonable to conclude that in a larger system these trends would continue. The merging of magnetic islands allows the islands to maintain a high aspect ratio, which maintains the magnetic tension necessary to drive reconnection. The steady reconnection rate allows for a constant rate of island growth, resulting in islands with a width that scales like the current sheet separation. These islands would be fully grown long before reaching the heliopause. The growth of these islands in the heliosheath is vital for the generation of anomalous cosmic rays (ACRs) by Fermi acceleration in islands (Drake et al. 2010). Since these islands are expected to be present in the sectored region, and the flux of ACRs is greatly reduced outside of the sectored region (Opher et al. 2011), both observations and models suggest that the sectored heliosheath has broken into magnetic islands.

Computations were performed at the National Energy Research Scientific Computing Center. This work has been supported by NSF grant ATM-0903964.

\section{REFERENCES}

Behannon, K. W.. Burlaga, L. F., Hoeksema, J. T., \& Klein, L. W. 1989, J. Geophys. Res., 94, 1245

Burlaga, L. F., Ness, N. F., \& Äcuna, M. H. 2007, Astrophys. J., 668,1246

Cassak, P., Shay, M. A., \& Drake, J. F. 2005, Phys. Rev. Lett., 95,235002

Drake, J. F., Opher, M., Swisdak, M., \& Chamoun, J. N. 2010, Astrophys. J., 709, 963

Harris, E. G. 1962, Nuovo Cim., 23, 115

Krimigis, S. M., Roelof, E. C., Decker, R. B., \& Hill, M. E. 2011, Nature, 474, 361

Opher, M., Drake, J. F., Swisdak, M., Schoeffler, K. M., Richardson, J. D., Decker, R. B., \& Toth, G. 2011, Astrophys. J., 734,71

Richardson, J. D., Kasper, J. C., Belcher, J. W., \& Lazarus, A. J. 2008, Nature, 454, 63
Schoeffler, K. M., Drake, J. F., \& Swisdak, M. 2011, Astrophys. J., 743,70

Schreier, R., Swisdak, M., Drake, J. F., \& Cassak, P. A. 2010, Phys. of Plas., 17, 110704

Shay, M. A., Drake, J. F., Rogers, B. N., \& Denton, R. E. 1999 Geophys. Res. Lett., 26, 2163

Shay, M. A., Drake, J. F., \& Swisdak, M. 2007, Phys. Rev. Lett., 99, 155002

Shay, M. A., Drake, J. F., Swisdak, M., Dorland, W., \& Rogers, B. N. 2003, Geophys. Res. Lett., 30,1345, doi:10.1029/2002GL016267

Smith, E. J. 2001, J. Geophys. Res., 106, 15,819

Yamada, M. 2007, Phys. Plasmas, 14, 058102 